\title{
Altered functional connectivity density in patients with herpes zoster and postherpetic neuralgia
}

This article was published in the following Dove Press journal:

Journal of Pain Research

\author{
Shunda Hongl,* \\ Lili Gu ${ }^{2}, *$ \\ Fuqing Zhou' \\ Jiaqi Liu' \\ Muhua Huang' \\ Jian Jiang' \\ Laichang $\mathrm{He}^{\prime}$ \\ Honghan Gong' \\ Xianjun Zeng'
}

'Department of Radiology, The First Affiliated Hospital, Nanchang University, Nanchang, Jiangxi, People's Republic of China; ${ }^{2}$ Department of Pain, The First Affiliated Hospital, Nanchang University, Nanchang, Jiangxi, People's Republic of China

*These authors contributed equally to this work
Correspondence: Jian Jiang Department of Radiology, The First Affiliated Hospital, Nanchang University, 17 Yongwaizheng Street, Nanchang, Jiangxi 330006, People's Republic of China

Tel +86 79l 88693825

Email jij2002cn@I26.com
Purpose: The aim of this study was to explore intrinsic functional connectivity patterns in patients with herpes zoster (HZ) and postherpetic neuralgia (PHN).

Patients and methods: Thirty-three right-handed HZ patients (13 males; mean age $57.15 \pm 9.30$ years), 22 right-handed $\mathrm{PHN}$ patients ( 9 males; mean age $66.13 \pm 6.77$ years), and 28 well-matched healthy controls (HC) (9 males; mean age 54.21 \pm 7.72 years) underwent resting-state functional magnetic resonance imaging for intrinsic functional connectivity analyses. Functional connectivity density (FCD) was calculated and compared among the PHN, HZ, and HC groups. In addition, the Pearson correlation coefficient was calculated to compare various clinical indices in the regions with abnormal FCD values.

Results: Compared with the HC, both $\mathrm{HZ}$ and PHN patients showed significantly decreased FCD in the precuneus, and patients with HZ displayed significantly increased FCD in the brainstem/ limbic lobe/parahippocampalgyrus, whereas patients with PHN displayed significantly increased FCD in the hippocampus (correlation thresholds $r=0.25$, voxel level of $P<0.01$ and Gaussian random field theory at a cluster level of $P<0.05$ ). However, the FCD was not significantly different between the PHN and HZ patients. Furthermore, the decreased FCD in the precuneus was positively correlated with the visual analog scale score in the PHN group ( $r=0.672 ; P=0.001)$. Conclusion: Decreased connectivity of the precuneus occurred in both $\mathrm{HZ}$ and PHN patients, indicating a disrupted default-mode network. Furthermore, in the HZ group (initial stage of the virus infection), hyperconnectivity was observed in systems involved in pain transmission and interpretation, but hyperconnectivity only occurred in the hippocampus in the PHN group (neuropathic pain stage).

Keywords: functional connectivity density, herpes zoster, postherpetic neuralgia, functional magnetic resonance imaging, resting state, pain

\section{Introduction}

Herpes zoster (HZ) is caused by reactivation of the latent varicella zoster virus (VZV) in the spinal or cranial sensory ganglia. It is characterized by a painful erythematous rash in the affected dermatome. ${ }^{1}$ Individuals infected with VZV who are later diagnosed with $\mathrm{HZ}$ are at high risk for progressing to postherpetic neuralgia (PHN). There is no precise consensus on the definition of PHN. Three alternative definitions are usually used by researchers: pain after the rash has healed over; ${ }^{2}$ persistent pain for more than 1 month after the zoster rash; ;,4 persistent pain for more than 3 month after the zoster rash. ${ }^{5} \mathrm{PHN}$ is the most common and persistent complication of chronic neuropathic pain caused by VZV and affects the peripheral and central nervous systems. ${ }^{6-8}$ The sharp pain experienced by patients with PHN seriously affects their quality of life ${ }^{9}$ and increases the economic burden on society. ${ }^{10}$ 
Recently, several neuroimaging studies ${ }^{1-15}$ have reported structural and functional alterations in PHN patients, including anatomical damage in the bilateral insula and superior temporal gyrus; ${ }^{11}$ increased cerebral blood flow in the $\mathrm{S} 1$ area, inferior parietal lobule, insula, thalamus, amygdala, and striatum; decreased cerebral blood flow in the frontal cortex; ${ }^{12}$ and abnormal activation or intrinsic activity in several regions. In task functional magnetic resonance imaging (fMRI), abnormal functional activation was observed in areas involved in affective response, sensory discrimination (the thalamus, primary and secondary somatosensory cortices, insula, and anterior cingulate cortex), and emotion and reward (ventral striatum, amygdala, and orbital frontal cortex). ${ }^{13-15}$ Unlike task-fMRI, resting-state fMRI (rs-fMRI) focuses on intrinsic functional segregation (local) and integration (distributed) to provide a view of the functional architecture underlying potential neural mechanisms of neuropathic pain. ${ }^{16}$ Regional homogeneity (ReHo) and the fractional aptitude of lowfrequency fluctuation (fALFF) are the most common and widely used methods for characterizing the dynamic properties of the neuronal processing unit. In patients with PHN, ReHo and fALFF show increased activity in the thalamus, cerebellum, limbic system, and frontal lobe and decreased activity in the temporal and parietal lobes. ${ }^{17}$ Cao et a $1{ }^{18}$ noted differences in local brain activity between $\mathrm{HZ}$ and PHN patients using the ReHo and fALFF. In contrast to patients with PHN, patients with $\mathrm{HZ}$ showed significantly increased activity in the limbic system, temporal lobe, occipital lobe, and parietal lobe and decreased activity in most of the cerebellum. ${ }^{18}$ Decreased homotopic connectivity in the dorsolateral prefrontal cortex, the precuneus and posterior cingulate cortex resulted in a decrease in functional integration. ${ }^{16}$ However, researchers have not determined whether connectivity network disruptions develop in patients with $\mathrm{HZ}$ and PHN. Thus, the aim of the current study was to provide a comprehensive view of the effects of $\mathrm{HZ}$ and PHN on organizational principles or the connectome of brain function. Functional connectivity density (FCD) is a graph-based and data-driven measurement defined as the number of functional binary network connectivities between each voxel throughout the brain. In contrast to seed-based functional connectivity and independent component analyses, the FCD algorithm has the advantage of exploring the whole-brain connectivity in an unbiased manner, ${ }^{19}$ as shown in several other studies. ${ }^{20-22}$

Multiple systems throughout the brain participate in the perception of pain; however, researchers have not determined how the whole brain functionally interacts and integrates during resting states in patients with $\mathrm{HZ}$ and PHN. In this study, we sought to evaluate the alterations in whole-brain FCD in patients with $\mathrm{HZ}$ and $\mathrm{PHN}$. We hypothesized that patients with PHN and HZ exhibit impaired intrinsic FCD throughout the brain, which was assessed using FCD analyses.

\section{Materials and methods Participants}

All participants were right-handed. Patients were recruited from The First Affiliated Hospital of Nanchang University. Two consulting physicians from the Pain Department made the clinical diagnosis of $\mathrm{HZ}$ and PHN based on the International Association for the Study of Pain criteria. ${ }^{23}$ Consistent with prior literature, all patients with $\mathrm{PHN}$ reported persistent pain for more than 30 days following the initial rash caused by $\mathrm{HZ}$ or after the resolution of the dermatological symptoms of HZ. ${ }^{3,4}$ It was a cross-sectional study, not a longitudinal study of the same patients. The HZ or PHN patients underwent MRI scanning after diagnosis and enrolled in the study within 24 hours. All participants underwent an evaluation using the visual analog scale (VAS) for spontaneous pain intensity checking prior to undergoing MRI scans. All patients reported VAS scores $\geq 5$, indicating moderate-to-severe pain ( 0 indicates no pain and 10 indicates the worst imaginable pain). No antidepressants or antipsychotic drugs were taken before the MRI scans. None of the participants had a history of psychiatric or neurological disorders or any other kind of pain. All age- and gender-matched healthy controls (HC) were also pain-free and did not have any structural abnormalities in the brain or neuropsychiatric disorders. Written informed consent was obtained from each participant or the participant's guardian prior to data acquisition. The current study was conducted according to the approved guidelines and in compliance with the principles of the Declaration of Helsinki. This study was approved by the Medical Research Ethics Committee and the Institutional Review Board of The First Affiliated Hospital of Nanchang University.

\section{MRI acquisition}

All participants underwent MRI scans using a 3.0T Siemens Trio TIM Scanner (Erlangen, Bavaria, Germany) at The First Affiliated Hospital of Nanchang University. The rs-fMRI data were obtained using an echo planar imaging sequence (repetition time $[\mathrm{TR}]=2,000 \mathrm{~ms}$, echo time $[\mathrm{TE}]=30 \mathrm{~ms}$, flip angle $=90^{\circ}$, matrix $=64 \times 64$, field of view $[\mathrm{FOV}]=220 \times 220$ $\mathrm{mm}, 4 \mathrm{~mm}$ slice thickness, 30 interleaved axial slices, and 240 time point acquisitions). High-resolution anatomic 3-D $\mathrm{T} 1$ ( $\mathrm{TR}=1,900 \mathrm{~ms}, \mathrm{TE}=2.26 \mathrm{~ms}$, flip angle $=9^{\circ}$, matrix $=240 \times 256, \mathrm{FOV}=215 \times 230 \mathrm{~mm}$, slice thickness $=1.0 \mathrm{~mm}$, 
and 176 sagittal slices) images, conventional T1-weighted ( $\mathrm{TR}=250 \mathrm{~ms}, \mathrm{TE}=2.46 \mathrm{~ms}$, slices $=19$, slice thickness $=5$ $\mathrm{mm}$, gap $=1.5 \mathrm{~mm}$, and FOV $=220 \times 220 \mathrm{~mm}$ ) images, and T2-weighted (TR $=4,000 \mathrm{~ms}, \mathrm{TE}=113 \mathrm{~ms}$, slices $=19$, slice thickness $=5 \mathrm{~mm}$, gap $=1.5 \mathrm{~mm}$, and FOV $=220 \times 220 \mathrm{~mm}$ ) images were also acquired. For rs-fMRI scans, participants were instructed to close their eyes, remain awake and clear their minds as much as possible. Foam pads were used to reduce head movements, and earplugs were used to minimize scanner noise during the MRI scan. After the scan, subjects were asked whether they had remained awake during the entire scan.

\section{Resting-state date preprocessing}

Before preprocessing, conventional T1-weighted and T2-weighted images were reviewed by two senior radiologists in the Department of Radiology of The First Affiliated Hospital of Nanchang University to rule out structural brain lesions. The rs-fMRI images were preprocessed to adjust the time series of images using a toolbox for Data Processing \& Analysis of Brain Imaging ${ }^{24}$ (DPABI 2.1; Chinese Academy of Sciences, Beijing, People's Republic of China) on MATLAB 7.14.0 (Mathworks, Natick, MA, USA). Preprocessing comprised the following steps: 1) removal of the first 10 functional volumes; 2) slice timing correction; 3) 3-dimensional motion correction; 4) coregistration of each individual's structural images to the functional images using a linear transformation. The transformed T1 structural images were segmented into white matter, gray matter, and cerebrospinal fluid using a new segment algorithm in DARTEL (diffeomorphic anatomical registration through exponentiated lie algebra); ${ }^{25} 5$ ) spatial normalization using the Montreal Neurological Institute template and resampling to $3 \times 3 \times 3 \mathrm{~mm}$ voxels; 6 ) linear detrending and nuisance signal removal (white matter, cerebrospinal fluid, global signal, 6-head motion parameters, 6-head motion parameters at one time point earlier, and the 12 corresponding squared items (Friston 24-parameter model) as covariates) via multiple regressions; and 7) bandpass filtering (ranging from 0.01 to $0.08 \mathrm{~Hz}$ ) to reduce the effects of low-frequency drift and high-frequency noise. In this study, we excluded four subjects according to the head motion criteria of a maximum spin $(x, y, z)$ of $<2.0^{\circ}$ and a maximum cardinal direction displacement $(x, y, z)$ of $<2.0 \mathrm{~mm}$. The group differences in head motion were evaluated among the patients $(\mathrm{HZ}, \mathrm{n}=33$ and PHN, $n=22)$ and the $\mathrm{HC}$ subjects $(\mathrm{n}=28)$ according to the frame-wise displacement criteria based on the method of Van Dijk et al. ${ }^{26}$

\section{FCD analysis}

FCD was used to evaluate the voxel-wise whole-brain functional connectivity. ${ }^{22}$ In this study, whole-brain FCD mapping was derived from the binary degree centrality data sets, ${ }^{27}$ which were calculated using DPABI (http://www. rfmri.org/dpabi). In a binary brain network, FCD is defined as the number of functional connections of a given voxel, as determined using the Pearson correlation coefficients between the time course of each voxel and those of other voxels using a threshold coefficient. To explore whether different thresholds had an impact on the results, five different correlation thresholds $(r=0.15,0.20,0.25,0.30,0.35)$ were computed in this study. ${ }^{28}$

\section{Statistical analysis}

SPSS 19.0 (SPSS Inc, Chicago, IL, USA) was used to analyze demographic and clinical data. Two-sample $t$-tests were performed to detect differences in age. The $\chi^{2}$ test was used to compare the gender ratio. The significance level was set to $P<0.05$.

SPM12 was used to smooth FCD maps with an 8-mm kernel and analyze the smoothed connectivity maps at the group level. Analysis of covariance (ANCOVA) was performed using DPABI toolbox to examine differences among the three groups ${ }^{19}$ and Bonferroni's corrections were used for multiple comparisons, after eliminating the effects of age, gender, and educational level by regression. The significance threshold correction was based on the Gaussian random field theory, with a voxel level of $P<0.01$ and a cluster level of $P<0.05$.

FCD values for brain regions that showed significant group differences were extracted and averaged. Correlational analyses were performed to compare the FCD values to the VAS and disease duration in each patient group. Pearson's correlation coefficients were calculated with a significance level of $P<0.05$.

\section{Results \\ Demographic and clinical characteristics}

Four patients were excluded due to head movements, and one participant in the HC group was excluded due to a history of mild traumatic brain injury. The final sample included 55 patients (33 HZ and $22 \mathrm{PHN}$ ) and $28 \mathrm{HC}$. The clinical characteristics of the $\mathrm{HZ}$ and PHN patients are listed in Table 1. No significant differences in age $(P=0.962,0.223,0.192$ for $\mathrm{PHN}$ vs $\mathrm{HC}, \mathrm{HZ}$ vs $\mathrm{HC}$, and $\mathrm{PHN}$ vs $\mathrm{HZ}$, respectively, two-sample $t$-test $)$ and gender $(P=0.522,0.557$, and 0.911 for PHN vs HC, HZ vs HC, and PHNvs HZ, respectively, $\chi^{2}$ test) were observed among the three groups. 


\section{FCD results}

The results of the ANCOVA revealed differences in the FCD maps between the three groups. Using the ANCOVA based on DPABI, we identified three differences in the FCD results: PHN vs $\mathrm{HC}, \mathrm{HZ}$ vs $\mathrm{HC}$, and PHN vs HZ. Intergroup differences (Figure 1) were also remarkably similar, based on the different correlation thresholds $(r=0.15,0.2,0.25$, 0.3 , and 0.35 ). Therefore, we primarily reported the FCD results in a weighted graph when the correlation threshold was 0.25. Compared to HC, patients with PHN exhibited a significantly decreased FCD in the precuneus and a significantly increased FCD in the bilateral hippocampus (HIP) (Figure 2 and Table 2). Compared to HC, patients with $\mathrm{HZ}$ exhibited significantly decreased FCD in the left precuneus and significantly increased FCD in the brainstem, limbic lobe, and parahippocampal gyrus (BM/LL/PHG) (Figure 2 and Table 2). However, the FCD was not significantly different between the PHN and HZ groups.

\section{Correlation between clinical variables and FCD}

According to the correlation analyses, the FCD value for the precuneus was positively correlated with the VAS score of

Table I Participants' information

\begin{tabular}{llll}
\hline Clinical information & $\begin{array}{l}\text { HZ patients } \\
(\mathbf{n = 3 3})\end{array}$ & $\begin{array}{l}\text { PHN patients } \\
(\mathbf{n = 2 2})\end{array}$ & $\mathbf{H C}(\mathbf{n = 2 8})$ \\
\hline Age (years, mean \pm SD) & $57.15 \pm 9.30$ & $66.13 \pm 6.77$ & $54.21 \pm 7.72$ \\
Gender (male/female) & $13 / 20$ & $9 / 13$ & $9 / 19$ \\
VAS score (mean \pm SD) & $6.39 \pm 1.17$ & $7.36 \pm 0.95$ & $\mathrm{NA}$ \\
Disease duration (days, & $11.24 \pm 6.67$ & $60.22 \pm 41.24$ & $\mathrm{NA}$ \\
mean \pm SD) & & & \\
\hline
\end{tabular}

Abbreviations: $\mathrm{HC}$, healthy controls; $\mathrm{HZ}$, herpes zoster; NA, not applicable; $\mathrm{PHN}$, postherpetic neuralgia; VAS, visual analog scale. the PHN group ( $r=0.672 ; P=0.001$ ) (Figure 3 and Table 3 ). However, a significant correlation was not observed between increased FCD and other clinical variables.

\section{Discussion}

In the current study, we investigated alterations in intrinsic functional connectivity patterns in both $\mathrm{HZ}$ and $\mathrm{PHN}$ patients using FCD. The FCD was decreased in the precuneus in both $\mathrm{HZ}$ and $\mathrm{PHN}$ patients. Moreover, patients with $\mathrm{PHN}$ showed increased FCD in the HIP, whereas patients with HZ showed increased FCD in the BM/LL/PHG. No significant difference was detected between patients with $\mathrm{HZ}$ and patients with PHN. Thus, the impairment in FCD in the precuneus may be relevant to pain-related impairment, and the connectivity of the precuneus may be disrupted in patients with HZ (initial stage of the virus infection). Alterations in functional connectivity in the limbic system (LL and HIP) may also play an important role in the neuropathological mechanism of pain in patients with $\mathrm{HZ}$.

In our study, decreased FCD was detected in the precuneus. The precuneus is the major functional node of the default-mode network. The default-mode netwrok shows the greatest activity at baseline, but its activity is suspended or attenuated during attention-demanding cognitive tasks requiring a high degree of functional connectivity. ${ }^{29,30}$ The posterior cingulate cortex/precuneus remains highly active at rest and continuously gathers information from both the external and internal milieu, which is important for monitoring sensory information. ${ }^{29}$ Furthermore, the activity of the precuneus impacts the network connectivity of cortical and subcortical structures involved in processing highly integrated and associative information; the precuneus plays an essential role in the implementation of a wide range of higher-order cognitive

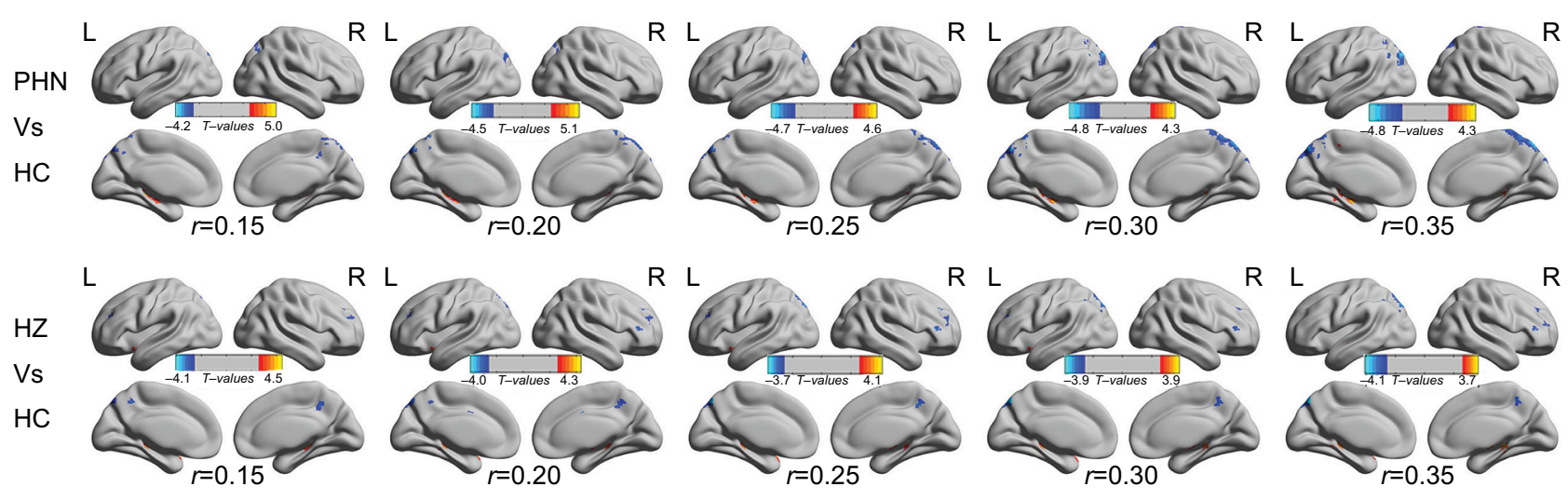

Figure I Intergroup difference in FCD according to different correlation thresholds.

Notes: Both comparisons of the PHN vs $\mathrm{HC}$ and $\mathrm{HZ}$ vs $\mathrm{HC}$ groups showed remarkably similar alterations in FCD in different brain areas according to different correlation thresholds ( $r=0.15,0.2,0.25,0.3$ and 0.35$)(P<0.00$ I, cluster $>20$ voxels, uncorrected). The hot (cool) color indicates brain areas with significantly increased (decreased) FCD. Abbreviations: $\mathrm{HZ}$, herpes zoster; $\mathrm{PHN}$, postherpetic neuralgia; $\mathrm{HC}$, healthy control; $\mathrm{FCD}$, functional connectivity density; $\mathrm{L}(\mathrm{R}) \mathrm{H}$, left (right) hemisphere. 

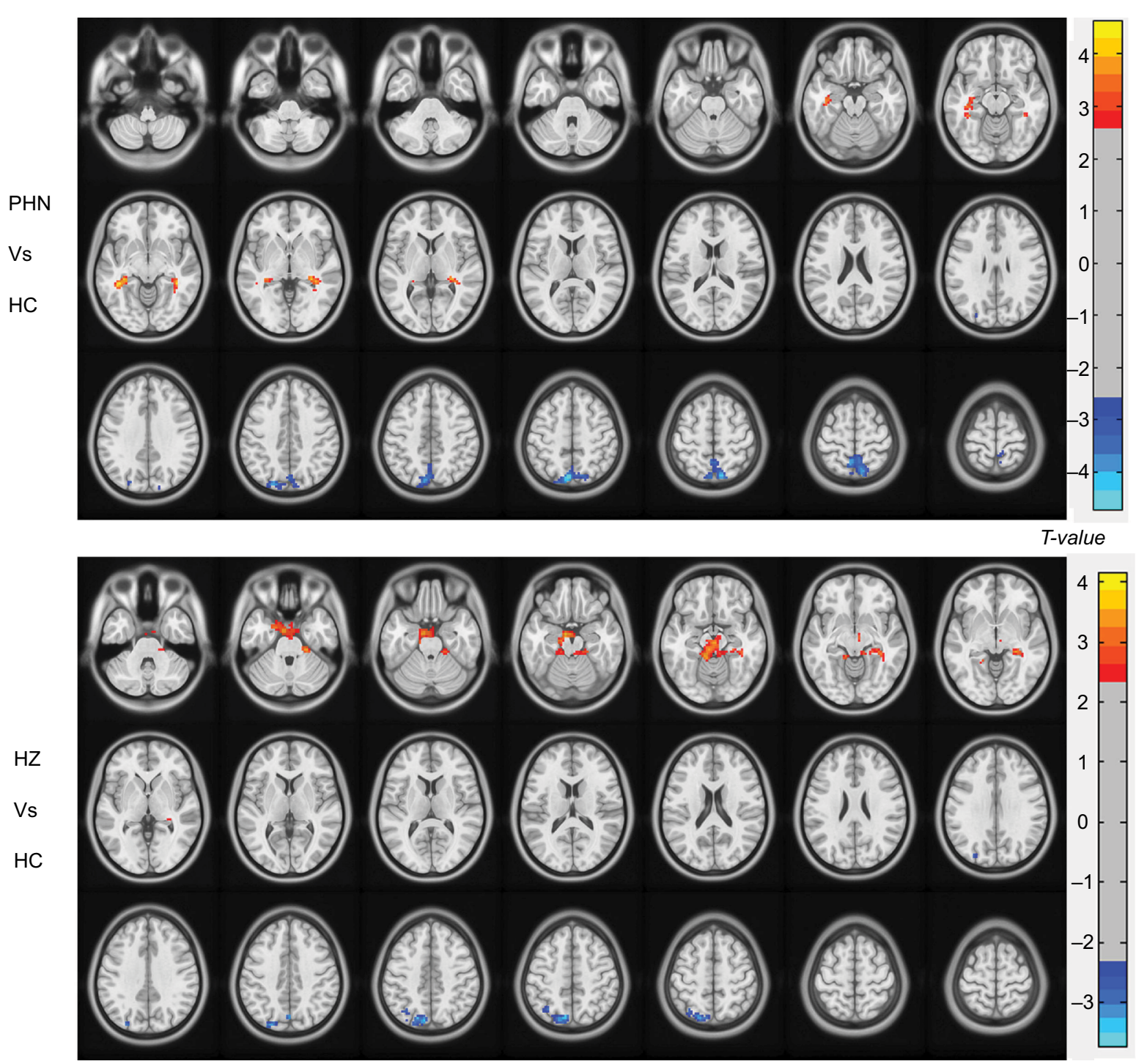

Figure 2 Between-group differences in FCD $(r=0.25)$ determined by ANCOVA (voxel level of $P<0.01$, GRF corrected at cluster level of $P<0.05$ ).

Abbreviations: $\mathrm{HZ}$, herpes zoster; PHN, postherpetic neuralgia; HC, healthy controls; FCD, functional connectivity density; ANCOVA, analysis of covariance; GRF, Gaussian random field.

Table 2 Significant differences in FCD among patients with $\mathrm{HZ}$ and $\mathrm{PHN}$ and $\mathrm{HC}(r=0.25)$

\begin{tabular}{llllll}
\hline Condition & L/R & $\begin{array}{l}\text { Brain } \\
\text { regions }\end{array}$ & $\begin{array}{l}\text { MNI } \\
\boldsymbol{X}, \mathbf{Y}, \mathbf{Z}\end{array}$ & Peak & $\begin{array}{l}\text { Voxel } \\
\text { size }\end{array}$ \\
\hline PHN vs HC & & & & & \\
& L/R & Precuneus & $-6,-75,51$ & -4.7079 & 462 \\
& L & HIP & $-39,-39,-12$ & 4.6489 & 128 \\
& R & HIP & $39,-39,-9$ & 4.0002 & 100 \\
HZ vs HC & & & & & \\
& L & Precuneus & $-9,-75,48$ & -3.6394 & 195 \\
& L/R & BM/LL/PHG & $-9,-9,-21$ & 3.7638 & 504 \\
\hline
\end{tabular}

Notes: ANCOVA (the results are based on GRF theory correction, with a voxe level of $P<0.01$ and a cluster level of $P<0.05$ ).

Abbreviations: ANCOVA, analysis of covariance; BM/LL/PHG, brainstem, limbic lobe and parahippocampal gyrus; FCD, functional connectivity density; GRF, Gaussian random field; HC, healthy control; HIP, hippocampus; HZ, herpes zoster; L(R), left (right) hemisphere; MNI, Montreal Neurological Institute; PHN, postherpetic neuralgia. functions. ${ }^{31}$ In this study, we observed decreased FCD in the precuneus in both $\mathrm{HZ}$ and PHN patients. Furthermore, a positive correlation between the VAS and the FCD value in the precuneus was observed. Based on these findings, the connectivity in the precuneus may be disrupted in patients with HZ (initial stage of the virus infection), and the impairment of the precuneus may be relevant to the sensation of pain. This impairment might also be related to the unpleasant cognitive experience of patients with $\mathrm{HZ}$.

In addition, we also observed significantly increased FCD in the limbic system and the brainstem of $\mathrm{HZ}$ patients. Compared to $\mathrm{HC}$, patients with $\mathrm{PHN}$ showed increased FCD in the HIP. Brain regions and functional networks have been shown to be altered during the interval between acute 


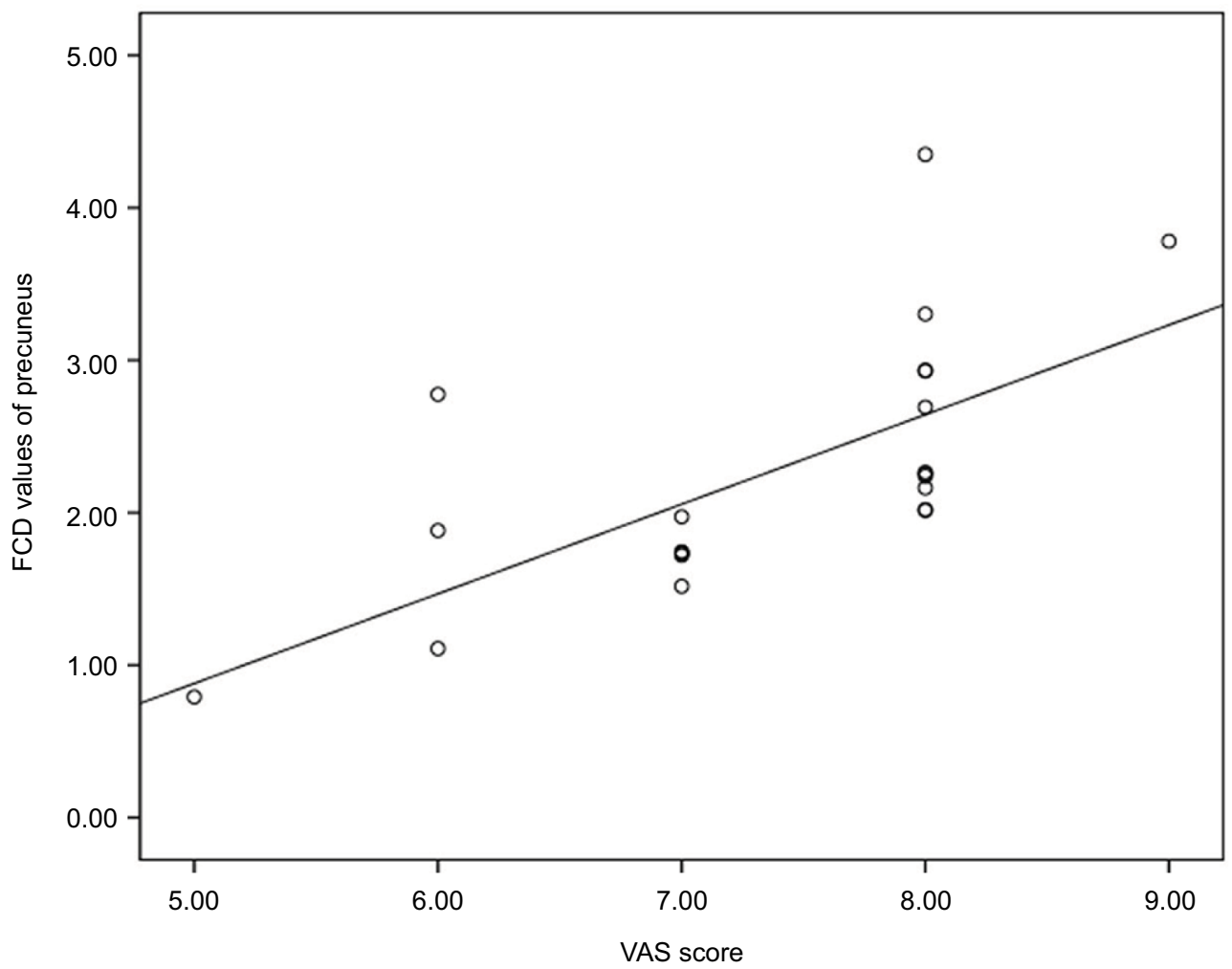

Figure 3 Correlations between abnormal FCD and VAS score in patients with PHN.

Notes: A significant positive correlation was observed between the FCD in the precuneus and the VAS in patients with PHN.

Abbreviations: VAS, visual analog scale; FCD, functional connectivity density; PHN, postherpetic neuralgi.

Table 3 Correlations between abnormal FCD and clinical variables

\begin{tabular}{lllllll}
\hline Clinical variables & PHN & & & HZ & \\
\cline { 2 - 3 } & Precuneus $(\boldsymbol{r} / \mathbf{P})$ & Hippocampus $(\boldsymbol{r} / \boldsymbol{P})$ & & Left precuneus $(\boldsymbol{r} / \mathbf{P})$ & BM/LL/PHG $(\boldsymbol{r} / \mathbf{P})$ \\
\hline VAS score & $0.672 / 0.001$ & $0.408 / 0.006$ & & $0.295 / 0.096$ & $0.133 / 0.459$ \\
Disease duration & $0.213 / 0.341$ & $0.208 / 0.352$ & & $0.159 / 0.377$ & $0.021 / 0.906$ \\
\hline
\end{tabular}

Abbreviations: BM/LL/PHG, brainstem/limbic lobe/parahippocampal gyrus; HZ, herpes zoster; PHN, postherpetic neuralgia; VAS, visual analog scale; FCD, functional connectivity density.

to chronic pain, ${ }^{32,33}$ and the HIP plays an important role in long-term pain, ${ }^{34-36}$ which involves pain-related memory and emotions. ${ }^{35,36}$ As shown in the study by Cardoso Cruz et $\mathrm{al},{ }^{37}$ the rat HIP is intimately involved in pain memories. This finding may explain why patients with PHN experience recurrent, long-term pain stimuli. We believe that unpleasant emotions are processed in the HIP and sent to the cerebral cortex to be transformed into long-term memory. In parallel, compared to $\mathrm{HC}$, patients with $\mathrm{HZ}$ showed significantly increased FCD in the $\mathrm{BM} / \mathrm{LL} / \mathrm{PHG}$. The brainstem plays major roles in pain processing and the modulation of nociceptive input. It is an important structure in the modulation of both ascending and descending pain pathways. The spinal cord-thalamus pathway participates in pain transmission, the cortex and limbic system are involved in pain interpretation, and the spinal cord-thalamus-cortex-limbic system is a pathway for pain transmission and interpretation. ${ }^{38}$ In this study, the brainstem and limbic system showed increased FCD, indicating that the BM/LL/PHG, which is involved in the transmission and interpretation of pain, was activated in patients with HZ. The regions involved in pain transmission and interpretation were mainly activated in the initial stage of the virus infection (HZ). The limbic regions of the pain matrix encode emotional aspects of pain perception, and the primary sensory region encodes the intensity of the pain sensation. ${ }^{39,40}$ Recent pain studies in patients with PHN have also reported functional changes in the limbic regions. ${ }^{12,14}$ But our results were different from previous touch task-fMRI study ${ }^{13}$ in PHN, which may indicated the central nervous mechanism of PHN were different between the spontaneous pain and touch-induced pain. rs-fMRI focuses on intrinsic functional connection or correlated spontaneous activity, 
while task-fMRI main reflects persistent or recurrent patterns of evoked coactivation.

In this study, different altered pattern was detected in $\mathrm{HZ}$ and PHN, but no significant difference of FCD was detected between HZ and PHN brains in this study. First, the pain of $\mathrm{PHN}$ and $\mathrm{HZ}$ is thought to result from damage in a nerve by $\mathrm{HZ}$, but the damage causes nerves in the affected dermatomic area of the skin to send abnormal electrical signals to the brain in PHN. A key factor in the neural plasticity underlying neuropathic pain is altered gene expression in sensory dorsal root ganglia neurons. ${ }^{1}$ In recently studies, functional and structural change might be correlated with HZ-PHN chronification. ${ }^{41}$ One of the reasons for the not-significant difference of FCD between $\mathrm{HZ}$ and $\mathrm{PHN}$ is inhomogeneity functional alteration or alteration of above the correlation thresholds $(r=0.25)$ in those patients, the sample size (patients with $\mathrm{PHN}$ ), and rigorous statistical methods of FCD comparison.

Several limitations to this study should be considered. First, PHN is associated with depression and anxiety, ${ }^{42,43}$ which may influence intrinsic brain function. Although no patients with depression and anxiety were enrolled in the current study, we did not specifically perform cognitive and emotional evaluations. Second, the sample size (patients with PHN) was limited and the pain duration (mean 60.2 days) was relatively short. Although monitoring patients from the acute pain stage (HZ) to the chronic stage (PHN) is not easy, we believe it is an effective way to eliminate individual differences that may influence the reliability of the results. Third, FCD was used to compute the long-range and short-range FCD values for each voxel in the brain. Future studies should further explore the differences between long-range FCD and short-range FCD throughout the brain. Finally, patients all have different dermatome involvement. Although no literature show the impact of the distribution of skin lesions on HZ brain, different affected areas may be lead to potentially difference in HZ brain. These need further exploration.

\section{Conclusion}

This novel study used voxel-wise FCD to investigate alterations in intrinsic functional connectivity in patients with $\mathrm{HZ}$ and PHN. The disrupted connectivity in the precuneus in both HZ and PHN patients reflects changes in the BM/LL/ PHG that may mediate the sensation of pain. This pathway was mainly activated in patients with $\mathrm{HZ}$ (initial stage of the virus infection), whereas the HIP was activated in patients with PHN (neuropathic pain stage). The HIP may participate in the production of pain memory. These findings expand our understanding of the functional characteristics of $\mathrm{HZ}$ and may provide new insights that will improve our understanding of the dysfunctional alterations implicated in the pathophysiology of $\mathrm{HZ}$.

\section{Acknowledgment}

The study was supported by grants from the Natural Science Foundation of Jiangxi Province (grant no 2015ZBAB205021) and the Jiangxi Province Education Department Support Program (grant no GJJ160128).

\section{Author contributions}

Shunda Hong, Lili Gu, Fuqing Zhou, and Jian Jiang designed the study. Shunda Hong, Lili Gu, Jiaqi Liu, and Muhua Huang acquired the data. Shunda Hong, Fuqing Zhou, Jian Jiang, and Jiaqi Liu processed the neuroimaging data. Jian Jiang performed the statistical analyses. Shunda Hong, Lili Gu, Fuqing Zhou, Jiaqi Liu, Muhua Huang, Jian Jiang, Laichang He, Honghan Gong, and Xianjun Zeng wrote the initial draft. All authors contributed to data analysis, drafting, and critically revising the paper and agree to be accountable for all aspects of the work.

\section{Disclosure}

The authors report no conflicts of interest in this work.

\section{References}

1. Jeon YH. Herpes zoster and postherpetic neuralgia: practical consideration for prevention and treatment. Korean J Pain. 2015;28(3): 177-184.

2. Schmader K. Postherpetic neuralgia in immunocompetent elderly people. Vaccine. 1998;16(18):1768-1770.

3. Gauthier A, Breuer J, Carrington D, Martin M, Rémy V. Epidemiology and cost of herpes zoster and post-herpetic neuralgia in the United Kingdom. Epidemiol Infect. 2009;137(1):38-47.

4. Snedecor SJ, Sudharshan L, Cappelleri JC, et al. Systematic review and meta-analysis of pharmacological therapies for pain associated with postherpetic neuralgia and less common neuropathic conditions. Int $J$ Clin Pract. 2014;68(7):900-918.

5. Keating GM. Shingles (Herpes Zoster) vaccine (Zostavax(Âß)): a review in the prevention of Herpes Zoster and Postherpetic Neuralgia. Biodrugs. 2016;30(3):243-254.

6. Gan EY, Tian EA, Tey HL. Management of herpes zoster and postherpetic neuralgia. Am J Clin Dermatol. 2013;14(2):77-85.

7. Bennett GJ, Watson CP. Herpes zoster and postherpetic neuralgia: past, present and future. Pain Res Manag. 2009;14(4):275-282.

8. Oaklander AL. The density of remaining nerve endings in human skin with and without postherpetic neuralgia after shingles. Pain. 2001;92(1):139-145.

9. Pickering G, Gavazzi G, Gaillat J, Paccalin M, Bloch K, Bouhassira D. Is herpes zoster an additional complication in old age alongside comorbidity and multiple medications? Results of the post hoc analysis of the 12-month longitudinal prospective observational ARIZONA cohort study. BMJ Open. 2016;6:e09689.

10. Friesen KJ, Falk J, Alessi-Severini S, Chateau D, Bugden S. Price of pain: population-based cohort burden of disease analysis of medication cost of herpes zoster and postherpetic neuralgia. J Pain Res. 2016; 9:543. 
11. Yu B. Microstructural abnormalities in gray matter of patients with postherpetic neuralgia: a diffusional kurtosis imaging study. Pain Physician. 2016;19(4):E601-E611.

12. Liu J, Hao Y, Du M, et al. Quantitative cerebral blood flow mapping and functional connectivity of postherpetic neuralgia pain: a perfusion fMRI study. Pain. 2013;154(1):110-118.

13. Geha PY, Baliki MN, Wang X, Harden RN, Paice JA, Apkarian AV. Brain dynamics for perception of tactile allodynia (touch-induced pain) in postherpetic neuralgia. Pain. 2008;138(3):641-656.

14. Zhang Y, Liu J, Li L, et al. A study on small-world brain functional networks altered by postherpetic neuralgia. Magn Reson Imaging. 2014;32(4):359-365.

15. Geha PY, Baliki MN, Chialvo DR, Harden RN, Paice JA, Apkarian AV. Brain activity for spontaneous pain of postherpetic neuralgia and its modulation by lidocaine patch therapy. Pain. 2007;128(1): $88-100$.

16. Jian J, Gu L, Dan B, et al. Altered homotopic connectivity in postherpetic neuralgia: a resting state fMRI study. J Pain Res. 2016;9:877-886.

17. Cao S, Song G, Zhang Y, et al. Abnormal local brain activity beyond the pain matrix in postherpetic neuralgia patients: a resting-state functional MRI study. Pain Physician. 2017;20(2):E303.

18. Cao S, Li Y, Deng W, et al. Local brain activity differences between herpes zoster and postherpetic neuralgia patients: a resting-state functional MRI study. Pain Physician. 2017;20(5):E687.

19. Beucke JC, Sepulcre J, Talukdar T, et al. Abnormally high degree connectivity of the orbitofrontal cortex in obsessive-compulsive disorder. JAMA Psychiatry. 2013;70(6):619-629.

20. Zuo XN, Ehmke R, Mennes M, et al. Network centrality in the human functional connectome. Cereb Cortex. 2012;22(8):1862-1875.

21. Tomasi D, Volkow ND. Functional connectivity density mapping. Proc Natl Acad Sci USA. 2010;107:9885-9890.

22. Li H, Lan L, Yi S, et al. Abnormal intrinsic functional hubs in severe male obstructive sleep apnea: evidence from a voxel-wise degree centrality analysis. PLoS One. 2016;11(10):e0164031.

23. Merskey H, Bogduk N. Classification of Chronic Pain: Descriptions of Chronic Pain Syndromes and Definitions of Pain Terms. 2nd ed. Seattle, WA: International Association for the Study of Pain; 1994.

24. Yan CG, Wang XD, Zuo XN, Zang YF. DPABI: data processing \& analysis for (resting-state) brain imaging. Neuroinformatics. 2016;14(3): 339-351.

25. Goto M, Abe O, Aoki S, et al. Diffeomorphic anatomical registration through exponentiated lie algebra provides reduced effect of scanner for cortex volumetry with atlas-based method in healthy subjects. Neuroradiology. 2013;55(7):869-875.

26. Van Dijk KR, Sabuncu MR, Buckner RL. The influence of head motion on intrinsic functional connectivity MRI. Neuroimage. 2012;59(1): 431-438.
27. Buckner RL, Sepulcre J, Talukdar T, et al. Cortical hubs revealed by intrinsic functional connectivity: mapping, assessment of stability, and relation to Alzheimer's disease. J Neurosci. 2009;29(6):1860-1873.

28. Cai F, Lei G, Gong H, et al. Network centrality of resting-state fMRI in primary angle-closure glaucoma before and after surgery. PLoS One. 2015;10(10):e0141389.

29. Raichle ME, MacLeod AM, Snyder AZ, Powers WJ, Gusnard DA, Shulman GL. A default mode of brain function. Proc Natl Acad Sci USA. 2001;98(2):676-682.

30. Whitfield-Gabrieli S, Ford JM. Default mode network activity and connectivity in psychopathology. Annu Rev Clin Psychol. 2012;8:49-76.

31. Cavanna AE, Trimble MR. The precuneus: a review of its functional anatomy and behavioural correlates. Brain J Neurol. 2006;129(Pt 3):564.

32. Apkarian AV, Hashmi JA, Baliki MN. Pain and the brain: specificity and plasticity of the brain in clinical chronic pain. Pain. 2011;152:49-64.

33. Hashmi JA, Baliki MN, Huang L, et al. Shape shifting pain: chronification of back pain shifts brain representation from nociceptive to emotional circuits. Brain. 2013;136:2751-2768.

34. Gondo M, Moriguchi Y, Kodama N, et al. Daily physical complaints and hippocampal function: an fMRI study of pain modulation by anxiety. Neuroimage. 2012;63:1011-1019.

35. Ploghaus A, Narain C, Beckmann CF, et al. Exacerbation of pain by anxiety is associated with activity in a hippocampal network. Neuroscience. 2001;21:9896-9903.

36. Lang S, Kroll A, Lipinski SJ, et al. Context conditioning and extinction in humans: differential contribution of the hippocampus, amygdala and prefrontal cortex. Eur J Neurosci. 2009;29:823-832.

37. Cardoso-Cruz H, Lima D, Galhardo V. Impaired spatial memory performance in a rat model of neuropathic pain is associated with reduced hippocampus-prefrontal cortex connectivity. J Neurosci. 2013;33: $2465-2480$.

38. Krarup C. An update on electrophysiological studies in neuropathy. Curr Opin Neurol. 2003;16(5):603-612.

39. Price DD. Psychological and neural mechanisms of the affective dimension of pain. Science. 2000;288:1769-1772.

40. Rainville P, Duncan GH, Price DD, Carrier B, Bushnell MC. Pain affect encoded in human anterior cingulate but not somatosensory cortex. Science. 1997;277:968-971.

41. Cao S, Qin B, Zhang Y, et al. Herpes zoster chronification to postherpetic neuralgia induces brain activity and grey matter volume change. Am J Transl Res. 2018;10(1):184-199.

42. Sah DW, Ossipo MH, Porreca F. Neurotrophic factors as novel therapeutics for neuropathic pain. Nat Rev Drug Discov. 2003;2:460-472.

43. Denkinger MD, Lukas A, Nikolaus T, Peter R, Franke S, Group AS. Multisite pain, pain frequency and pain severity are associated with depression in older adults: results from the ActiFE Ulm study. Age Ageing. 2014;43:510-514.
Journal of Pain Research

\section{Publish your work in this journal}

The Journal of Pain Research is an international, peer reviewed, open access, online journal that welcomes laboratory and clinical findings in the fields of pain research and the prevention and management of pain. Original research, reviews, symposium reports, hypothesis formation and commentaries are all considered for publication.

\section{Dovepress}

The manuscript management system is completely online and includes a very quick and fair peer-review system, which is all easy to use. Visit http://www.dovepress.com/testimonials.php to read real quotes from published authors. 